\title{
Conditional Associative Memory for Musical Stimuli in Nonmusicians: Implications for Absolute Pitch
}

\author{
Patrick Bermudez and Robert J. Zatorre \\ Montréal Neurological Institute, McGill University, Montréal, Québec, Canada H3A 2B4
}

A previous positron emission tomography (PET) study of musicians with and without absolute pitch put forth the hypothesis that the posterior dorsolateral prefrontal cortex is involved in the conditional associative aspect of the identification of a pitch. In the work presented here, we tested this hypothesis by training eight nonmusicians to associate each of four different complex musical sounds (triad chords) with an arbitrary number in a task designed to have limited analogy to absolute-pitch identification. Each subject underwent a functional magnetic resonance imaging scanning procedure both before and after training. Active condition (identification of chords)-control condition (amplitude-matched noise bursts) comparisons for the pretraining scan showed no significant activation maxima. The same comparison for the posttraining scan revealed significant peaks of activation in posterior dorsolateral prefrontal, ventrolateral prefrontal, and parietal areas. A conjunction analysis was performed to show that the posterior dorsolateral prefrontal activity in this study is similar to that observed in the aforementioned PET study. We conclude that the posterior dorsolateral prefrontal cortex is selectively involved in the conditional association aspect of our task, as it is in the attribution of a verbal label to a note by absolute-pitch musicians.

Key words: frontal cortex; auditory; memory; conditional; music; absolute pitch

\section{Introduction}

Absolute or, as it is commonly referred to, perfect pitch is generally defined as the ability to identify the names of musical pitches without reference to a standard (Bachem, 1937; Ward, 1999). Although it seems likely that early and intensive musical training is necessary to develop absolute pitch (AP), these do not seem to be sufficient conditions, and the factors, genetic or otherwise, that interact with this training to dispose the individual to the acquisition of AP are still very much in question (Takeuchi and Hulse, 1993; Baharloo et al., 1998; Zatorre, 2003). A considerable amount of research has delved into various behavioral aspects of AP (Takeuchi and Hulse, 1993; Ward, 1999), but relatively few studies have investigated its neural correlates (Klein et al., 1984; Hantz et al., 1992; Wayman et al., 1992; Zatorre et al., 1998; Keenan et al., 2001). Electrophysiological studies using eventrelated potential techniques (Hantz et al., 1992; Wayman et al., 1992), for instance, have mainly shown a reduced P300 component among AP musicians in auditory oddball paradigms.

The scientific interest of AP lies in the fact that performing a relatively simple AP task, such as hearing a note in isolation and giving its name, represents a fairly well circumscribed complex cognitive task that can lend insight into various aspects of cognitive processing. We can describe some of the components in the

Received April 20, 2005; revised June 28, 2005; accepted July 6, 2005.

This work was supported by the International Foundation for Music Research and the Canadian Institutes of Health Research. We thank Marc Bouffard for his expertise and assistance, Penelope Kostopoulos and Michael Petrides for kind consultations, as well as the staff of the McConnell Brain Imaging Centre.

Correspondence should be addressed to Patrick Bermudez, Department of Neuropsychology, Room 276, Montréal Neurological Institute, Montréal, Québec, Canada H3A 2B4. E-mail: pat@ego.psych.mcgill.ca.

DOI:10.1523/JNEUROSCI.1560-05.2005

Copyright $\odot 2005$ Society for Neuroscience $\quad$ 0270-6474/05/257718-06\$15.00/0 chain of processing needed to accomplish an AP task without understanding how the ability is acquired. In a positron emission tomography (PET) study by Zatorre et al. (1998), both AP and non-AP musicians listened passively to single notes and, in a separate condition, identified ascending or descending major or minor thirds. Activation in the left posterior dorsolateral frontal (DLF) cortex for AP possessors when identifying single notes, and nonpossessors when identifying the interval between two notes, was hypothesized to be a reflection of the ability to retrieve an arbitrary conditional association between a stimulus attribute (pitch or interval value) and a verbal label (note or interval name). This interpretation is based on an accrual of evidence from human lesion studies (Petrides, 1990, 1997), monkey lesion work (Stamm, 1973; Podbros et al., 1980; Petrides, 1986), and functional brain imaging in healthy individuals (Petrides et al., 1993b; Owen et al., 1996; Lepage et al., 2003; Boettiger and D'Esposito, 2005) concerning fine distinctions in the behavioral roles ascribed to different areas of the DLF cortex. Whereas the mid-DLF cortex seems to be involved in modality-independent monitoring of information in working memory, the posterior DLF cortex seems crucial in the conditional aspect of associations in which there are competing possible responses to various stimuli.

The experiment presented here sought to test the post hoc hypothesis of Zatorre et al. (1998) that the posterior DLF cortex is involved in the conditional association between an auditory stimulus and an arbitrary label. We designed a task intended to have limited analogy to AP identification for musically naive subjects and a functional magnetic resonance imaging (fMRI) protocol to detect the predicted involvement of the posterior DLF in the performance of this task. 


\section{Materials and Methods}

Subjects. Subjects were eight normal, right-handed volunteers (six women, two men; mean age, 26.1 years) from the working and studying environment of the Montreal Neurological Institute (MNI) at McGill University. None had formal musical knowledge that would allow them to conventionally label the experimental stimuli or have preexisting associations for them.

Stimuli. Stimuli consisted of minor, augmented, diminished, and major triads constructed with piano timbres on a $\mathrm{C}_{4}$ root and lasting $2 \mathrm{~s}$ each, as well as bursts of white noise with temporal envelopes matched to the chordal stimuli.

Experimental task and training. Subjects took part in two fMRI scanning sessions, one before and one after training. There were two training sessions outside the scanner, the first $2 \mathrm{~d}$ before and the second $1.5 \mathrm{~h}$ before the posttraining scan. During this training, subjects learned to associate each of the four chordal stimuli described above with one of four numbers (1-4). These chords were presented binaurally through dynamic headphones in a fixed order, along with their associated numerals appearing on a computer screen (20 trials; five repetitions of each chord). A recall test was then performed during which the chords were presented in a randomized order (40 trials; 10 repetitions of each chord). Subjects responded by identifying the chord with a button press of the right hand on a custom four-button response box and were given feedback after incorrect responses by hearing the stimulus again and seeing its associated numeral on the screen. This process of learning and recall was repeated five times in each of two training sessions.

Scanning. Scanning was performed on a Siemens (Erlangen, Germany) Vision $1.5 \mathrm{~T}$ magnetic resonance imaging scanner using a temporally sparse volume acquisition (Belin et al., 1999) in a block design. This entailed two runs of 96 volume acquisitions at 10 s intervals with stimulus presentations grouped into $80 \mathrm{~s}$ blocks. The "on" blocks contained auditory stimulation, either chords or acoustically matched noise bursts, whereas the "off" intervals served as a silent baseline. The 20 slices comprising the functional volume were acquired $5 \mathrm{~s}$ after each stimulus onset. A T1-weighted three-dimensional volume was acquired for anatomical localization for each subject [echo time (TE), $9.2 \mathrm{~ms}$; repetition time (TR), $22 \mathrm{~ms}$; matrix size, $256 \times 256$; voxel size, $1 \times 1 \times 1 \mathrm{~mm}]$. A functional volume was then defined, consisting of 20 contiguous $5-\mathrm{mm}$ thick axial T2* gradient echo-planar images (TE, $50 \mathrm{~ms}$; TR, $10 \mathrm{~s}$; matrix size, $64 \times 64$; voxel size, $5 \times 5 \times 5 \mathrm{~mm}$ ) aligned in plane with the Sylvian fissure.

During the active condition of the posttraining fMRI session, subjects heard one chord every $10 \mathrm{~s}$ presented binaurally via pneumatic earphones and identified it with a button press, as in the recall condition during training (with the exception of feedback). For the pretraining fMRI session, subjects were instructed to give an arbitrary button press to each stimulus, because they had not yet acquired the means to respond meaningfully. During the control condition, which served as a basic auditory stimulation and sensorimotor control, subjects heard the noise stimuli and gave an arbitrary button press. During silent periods, subjects simply lay at rest.

Analyses. Blood oxygenation level-dependent (BOLD) images were motion corrected and spatially smoothed with a $10 \mathrm{~mm}$ full-width at half-maximum Gaussian blurring kernel. Both functional and anatomical images were then linearly transformed into MNI stereotaxic space (Collins et al., 1994). Statistical analysis of the BOLD data was based on a general linear model with correlated errors (Worsley et al., 2002). The following three $t$ statistic subtraction images were generated for both the pretraining and posttraining fMRI sessions: (1) active condition (chords) - control condition (noise), (2) active condition (chords) silence condition, and (3) control condition (noise) - silence condition. The first of these, chords - noise, is the subtraction of interest that we shall dwell on, especially in the comparison between scanning sessions pretraining (S1) and posttraining (S2), therefore, $\mathrm{S}_{2}$ chords - noise - $\mathrm{S} 1_{\text {chords }}$ - noise. A minimum threshold for statistical significance for the subtractions and comparisons between pretraining and posttraining fMRI sessions was computed according to the random field theory (Worsley et al., 2002).

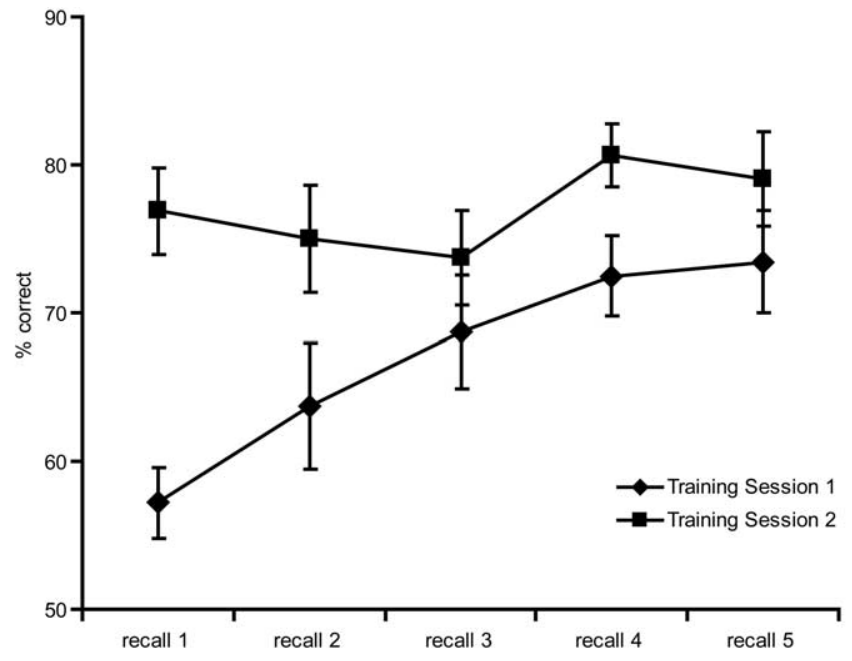

Figure 1. Average subject performance on the recall task in training sessions 1 and 2. Error bars indicate $S E$.

Additionally, we performed a variant of the conjunction analysis introduced by Price and Friston (1997) to determine which areas of activity were common to both the contrast of interest in this experiment and the tone perception condition among AP subjects in the PET experiment of Zatorre et al. (1998). Briefly, this consists of performing a logical "AND" operation on all suprathreshold voxels existing in both the $t$ statistic image of the tones - noise contrast in AP subjects from Zatorre et al. (1998) and the $t$ statistic image of the $\mathrm{S} 2_{\text {chords }}-$ noise $-S 1_{\text {chords }}$ - noise contrast in this study. This method is simple to implement and interpret and avoids some problems associated with the method of Price and Friston (1997) (Nichols et al., 2005). The voxel sizes for both $t$ statistic images are comparable (1.5-2 $\mathrm{mm}$ in each dimension), although the reconstruction and analysis of the PET data of Zatorre et al. (1998) used larger filtering than our fMRI data (18 mm Hanning vs $10 \mathrm{~mm}$ Gaussian, respectively).

\section{Results}

\section{Behavioral}

The average absolute correlation between stimulus and subject button-press for the pretraining fMRI session was $r=0.077$ (that is, essentially random). An ANOVA on the percentage of correct identification scores from the two training sessions revealed a significant session $\times$ recall interaction $(F=5.19 ; p=0.003)$ (Fig. $1)$. Subjects showed gradual and significant improvement during the first training session (first vs fifth recall test; $p<0.001$ ) and maintained stable performance throughout the second training session with an average no different from the highest scores achieved in the first $(p=0.41)$. Performance of the task during the posttraining scan was significantly weaker $(p<0.0001)$, likely attributable to a combination of more difficult performance conditions and relatively poorer sound quality (pneumatic transducers) compared with the laboratory training conditions. Also, whereas the response required of subjects during training was self-paced, responses not given within $4.5 \mathrm{~s}$ in the scanner were counted as errors. Despite this, in-scanner performance was significantly above chance (mean, $57 \%$; $p<0.001$ ).

\section{Imaging: contrasts}

A statistical threshold of $t=4.9$ was established using the abovedescribed methods. The noise - silence subtractions of both the S1 and S2 scans showed activity in primary and secondary auditory cortices related to the perception of the auditory stimuli, as well as motor and sensory activity related to the right-handed 


\begin{tabular}{|c|c|c|c|c|}
\hline & \multicolumn{3}{|c|}{$\begin{array}{l}\text { Stereotaxic coordinates } \\
\text { (MNI space) }\end{array}$} & \multirow[b]{2}{*}{$t$ value } \\
\hline & $x$ & $y$ & $z$ & \\
\hline \multicolumn{5}{|l|}{ Right hemisphere } \\
\hline Middle frontal gyrus (rostral area 8) & 44 & 34 & 18 & 5.13 \\
\hline Middle frontal gyrus (area 8) & 38 & 18 & 32 & 5.95 \\
\hline Middle frontal gyrus (area 8) & 42 & 14 & 36 & 5.99 \\
\hline \multicolumn{5}{|l|}{ Medial aspect of superior frontal gyrus } \\
\hline (area 8) & 4 & 26 & 40 & 5.18 \\
\hline \multicolumn{5}{|l|}{ Medial aspect of superior frontal gyrus } \\
\hline (pre-SMA) & 2 & 20 & 50 & 5.98 \\
\hline Middle frontal gyrus (rostral area 6) & 32 & -2 & 48 & 5.35 \\
\hline Middle frontal gyrus (rostral area 6) & 36 & -2 & 58 & 6.26 \\
\hline Mid-ventrolateral frontal (area 47/12) & 30 & 26 & 6 & 4.85 \\
\hline Mid-ventrolateral frontal (area 47/12) & 30 & 24 & 2 & 4.84 \\
\hline Angular gyrus (area 7) & 34 & -56 & 48 & 5.80 \\
\hline \multicolumn{5}{|l|}{ Left hemisphere } \\
\hline Middle frontal gyrus (rostral area 8) & -42 & 22 & 22 & 6.88 \\
\hline Inferior parietal lobe (area 7, near bor- & & & & \\
\hline $\begin{array}{l}\text { der with } 40 \text { ) } \\
\text { Inferior parietal lobe (area 7, near bor- }\end{array}$ & -32 & -52 & 42 & 5.42 \\
\hline der with 40 and 39) & -36 & -52 & 48 & 5.25 \\
\hline
\end{tabular}

SMA, Supplementary motor area.

response through the custom four-button response box. The chords - noise subtraction for the pretraining fMRI session $\left(\mathrm{S} 1_{\text {chords - noise }}\right)$ showed no suprathreshold activation other than bilateral minima in the auditory cortices, immediately posterior to Heschl's gyrus in the right hemisphere $(x=44, y=-26, z=14$; $t=-7.64)$ and more posteriorly in the planum temporale on the left $(x=-44, y=-36, z=16 ; t=-7.62)$. However, this same contrast in the posttraining fMRI session ( $\mathrm{S} 2_{\text {chords - noise }}$ ) showed a number of significant peaks, most notably the left and right posterior DLF cortices (cytoarchitectonic area 8) (Petrides and Pandya, 1994), right rostral area 6/caudal 8, bilateral midventrolateral frontal activations (area 47/12), and the inferior parietal lobes bilaterally (areas 7 and 40). We further contrasted the chords - noise subtraction between sessions $\left(\mathrm{S} 2_{\text {chords }}-\right.$ noise $-\mathrm{S} 1_{\text {chords }}$ - noise). This contrast yields essentially the same pattern of activation as $\mathrm{S} 2_{\text {chords - noise }}$ alone, and we will therefore focus on $S 2_{\text {chords }}$ - noise $-S 1_{\text {chords }}$ - noise henceforth, with the benefit of its direct pretraining/posttraining comparison (Table 1). Of note are the following: (1) a peak centered at the inferior portion of the left middle frontal gyrus, near the rostral border of area 8 and partly in the inferior frontal sulcus (Fig. 2a); (2) a band of activity along area 8 of the right middle frontal gyrus with at least two separate peaks (Fig. 2b,c); and (3) a peak centered at the junction of the right posterior middle frontal gyrus, the superior precentral sulcus, and the superior frontal sulcus, corresponding to rostral area $6 /$ caudal 8 (Figs. $2 d, 3$ ). The bilateral mid-ventrolateral activity reported for $\mathrm{S} 2$ chords - noise $(x=-36, y=-54, z=50 ; t=$ $6.50 ; x=30, y=24, z=2 ; t=6.41)$ is just below statistical threshold in the right hemisphere in the between-session contrast $(x=30, y=26, z=6 ; t=4.85)$.

\section{Imaging: conjunction}

A spatial conjunction between the tones - noise contrast in AP subjects from Zatorre et al. (1998) and the $\mathrm{S} 2$ chords - noise -

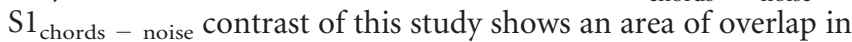
the left middle frontal gyrus, in the rostral portion of area 8 (Fig. $4 a$ ). It was noticed that the pattern of frontal lobe activity is primarily in the left hemisphere in the results of Zatorre et al. (1998), whereas our activity is more extensive in what seem to be

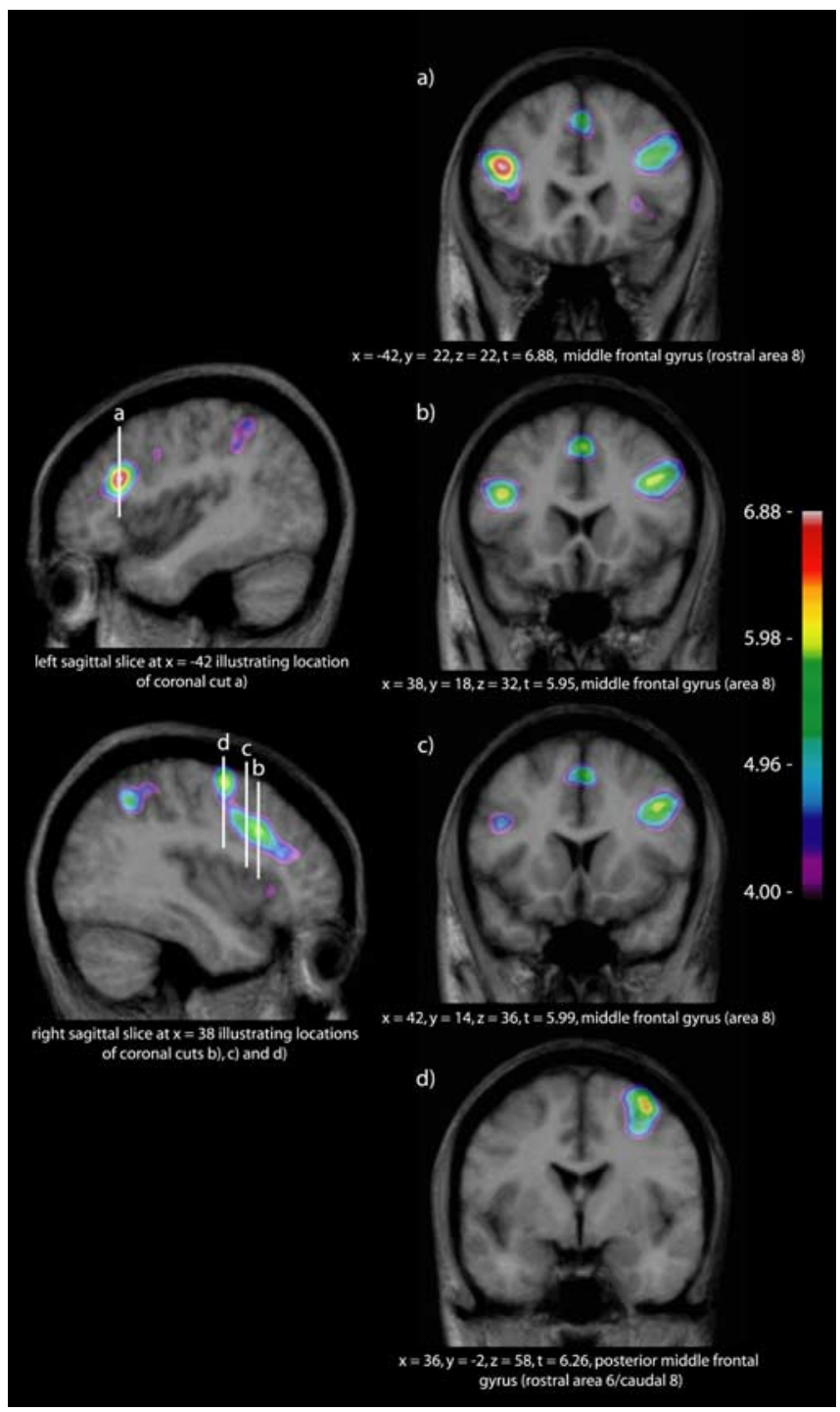

Figure 2. $S 2_{\text {chords }}$ - noise $-S 1_{\text {chords }}$ - noise contrast. The sagittal cuts to the left of the figure indicate the location of the coronal cuts to the right. $\boldsymbol{a}$, Inferior portion of the left middle frontal gyrus, near the rostral border of area 8 and partly in the inferior frontal sulcus. $\boldsymbol{b}, \boldsymbol{c}$, A band of activity along area 8 of the right middle frontal gyrus. $\boldsymbol{d}$, The junction of the right posterior middle frontal gyrus and superior precentral sulcus, corresponding to rostral area 6/caudal 8.

homologous areas of the right hemisphere. We verified this by "flipping" our results about $x=0$, thereby effectively creating a mirror image at the midsagittal line, and proceeded to use this image in a conjunction operation identical to that described above. This provides an additional common area more posteriorly and superiorly along area 8 of the middle frontal gyrus (Fig. $4 b)$.

\section{Discussion}

In this work, a hypothesis proposed by Zatorre et al. (1998) concerning the involvement of the posterior DLF in the conditional associative aspect of the note-naming ability of AP musicians was tested. This was done by having musically naive subjects learn to discriminate between four types of musical sounds and associate each to an arbitrary label. The data presented are concordant with the proposed hypothesis.

\section{Posterior dorsolateral frontal activations}

Data from a number of monkey, human lesion, and imaging studies are converging to suggest that posterior DLF regions cor- 


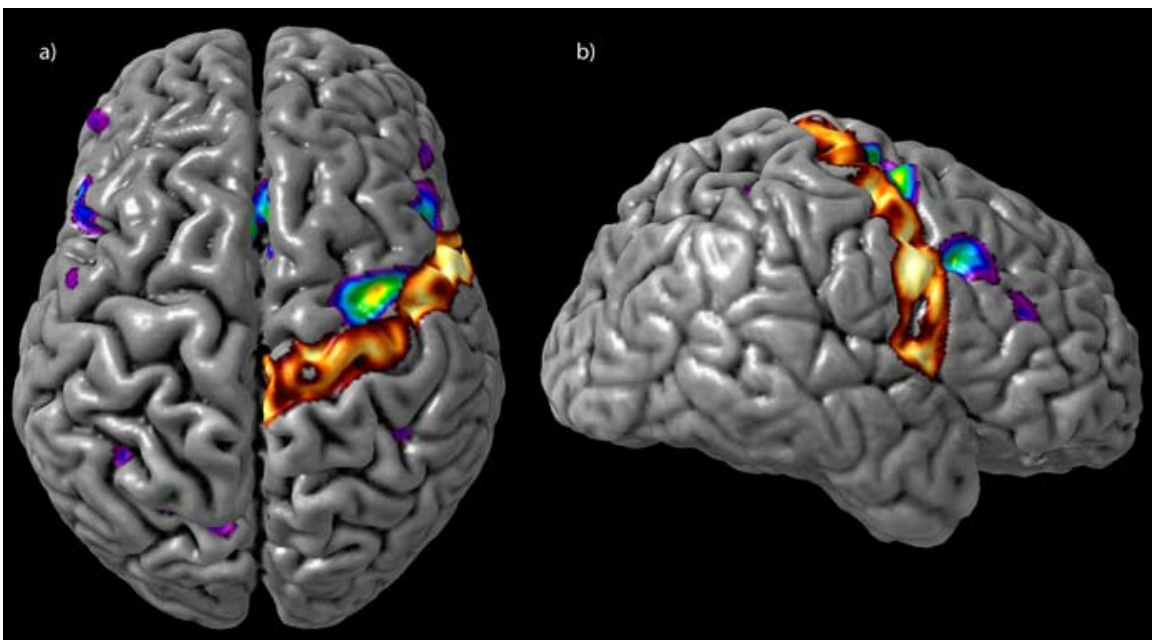

Figure 3. The $S 2_{\text {chords }- \text { noise }}-\mathrm{S} 1_{\text {chords - noise }}$ group contrast and a $40 \%$ thresholded probability map of the right precentral gyrus, derived from the MNI International Consortium for Brain Mapping 152 subject normal cohort (Mazziotta et al., 2001), are overlaid onto a three-dimensional rendering of a single subject. This clearly illustrates the position of the peak shown in Figure $2 d$ as lying anterior to the precentral gyrus in rostral area 6/caudal 8 .

responding to areas 8 and rostral 6 are involved in critical aspects of conditional associative memory (Halsband and Passingham, 1985; Petrides, 1985; Crowne et al., 1989; Halsband and Freund, 1990; Petrides et al., 1993a; Lepage et al., 2003). This type of memory is implicated in situations in which several alternative responses to different stimuli exist and a correct response must be provided when cued by the appropriate stimulus. This is to be distinguished from a simple stimulus-response association in which a response to a cue is extinguished in the absence of that cue. For instance, in a condition of a PET study in which subjects had to identify one of eight abstract designs previously associated with one of eight color cues, Petrides et al. (1993a) observed activity in the left caudal superior frontal sulcus, area 8 . In our results, activity in a similar area was somewhat more extensive in the right hemisphere, perhaps because of the tonal nature of the stimuli (Zatorre et al., 2002), but strongly bilateral nonetheless (Fig. 2).

Considerable controversy exists in the brain-imaging literature concerning results for conditional motor tasks, typically in the form of a specific motor act of the hand cued by either an auditory or visual stimulus (Deiber et al., 1997; Grafton et al., 1998; Kurata et al., 2000). Nearly all such studies elicit activity contralateral to the hand used and often more posteriorly in area 6. We call attention to the fact that our area 6 activity is rostral (Amiez et al., 2005) and ipsilateral to the hand used to respond in our task (Figs. $2 d, 3$ ). Now, to be clear, our task is not a strict motor conditional task. Given the way subjects were trained (see Materials and Methods, Experimental task and training), the conditional association is likely to have formed between a sound (chord heard through headphones) and numeral (1-4 seen on screen) or, more abstractly, sound and number. In our task, the way to indicate this correspondence of chord to number is indeed a motor response, but the initial association itself is not to the motor act. This perhaps accounts for part of the difference in observed results, but, more importantly, our data favor certain models that ascribe multimodal executive function to some of the frontal lobe areas implicated. It should also be noted that, despite the fact that this peak of activation overlaps with an area suspected to contain the frontal eye field (Paus, 1996; Rosano et al., 2003; Koyama et al., 2004; Pierrot-Deseilligny et al., 2004), there is no explicit visual involvement in our auditory conditional associative task.

The overlap between our data and those of Zatorre et al. (1998) in the posterior DLF cortex provides the strength of converging evidence (Fig. 4). In the task of Zatorre et al. (1998), a single tone selected from the range of $\mathrm{F}^{\#} 3$ to $\mathrm{C}^{\#} 5$ was presented and covertly named by musicians with AP. In our task, a chord was identified by nonmusicians who had learned to associate each of four chords with a number. Both of these tasks require the association of a stimulus dimension to an arbitrary label, the creation of an association where no intrinsic one exists.

Mid-ventrolateral prefrontal activations The mid-ventrolateral prefrontal cortex (area 47/12) is thought to be involved in the maintenance of memory components and active or selective retrieval among several encoded features of a stimulus or several possible responses to stimuli in situations in which the correct response is not immediately suggested by strong stimulus-to-stimulus associations (Kostopoulos and Petrides, 2003). Such active retrieval mechanisms are different from basic recognition memory. Activation of the mid-ventrolateral prefrontal cortex in this study was most obvious in the $\mathrm{S} 2$ chords - noise contrast and somewhat stronger in the right hemisphere, which, again, is consistent with the tonal nature of stimuli. The locations of our peaks are very similar to those reported by other researchers (Cadoret et al., 2001; Kostopoulos and Petrides, 2003) in studies in which a task required selective postencoding retrieval among various features of a stimulus.

\section{Temporal and parietal lobe activations}

An unpredicted but potentially interesting result is that of pretraining and posttraining differences in the activity of the auditory cortex, principally the auditory belt posterior to Heschl's gyrus (Penhune et al., 1996; Schneider et al., 2002). The pretraining contrast of chords - noise $\left(\mathrm{S} 1_{\text {chords }}\right.$ - noise $)$ showed significant minima bilaterally, whereas the same contrast posttraining $\left(\mathrm{S} 2_{\text {chords }}\right.$ - noise $)$ did not, suggesting a change in the cortical response to the stimuli after training. The results of session 1 may have been caused by the greater spectral composition of the white-noise control stimuli compared with the chord stimuli. Both stimulus types elicited less BOLD response in the second session and it would seem that the relative relationship of the stimuli to each other also changed. There is precedent for reduced auditory cortex response as a result of training and exposure (Cansino and Williamson, 1997; Morris et al., 1998; Jäncke et al., 2001; Teismann et al., 2004), and our data are likely another example of short-term plasticity in auditory cortices as a function of learning.

Bilateral parietal activity, specifically in the left angular gyrus and right inferior parietal lobule (areas 7 and 40, respectively), was also notable in the $\mathrm{S} 2_{\text {chords }}$ - noise and $\mathrm{S} 2_{\text {chords }}-$ noise $\mathrm{S} 1_{\text {chords }- \text { noise }}$ contrasts. These regions are frequently implicated, in concert with frontal areas, for aspects of directed and intentional action and response selection (Rizzolatti and Luppino, 2001; Rubia et al., 2001; Göbel et al., 2004), as well as the direction of attention to motor acts (Rushworth et al., 1997, 2001; Nobre, 


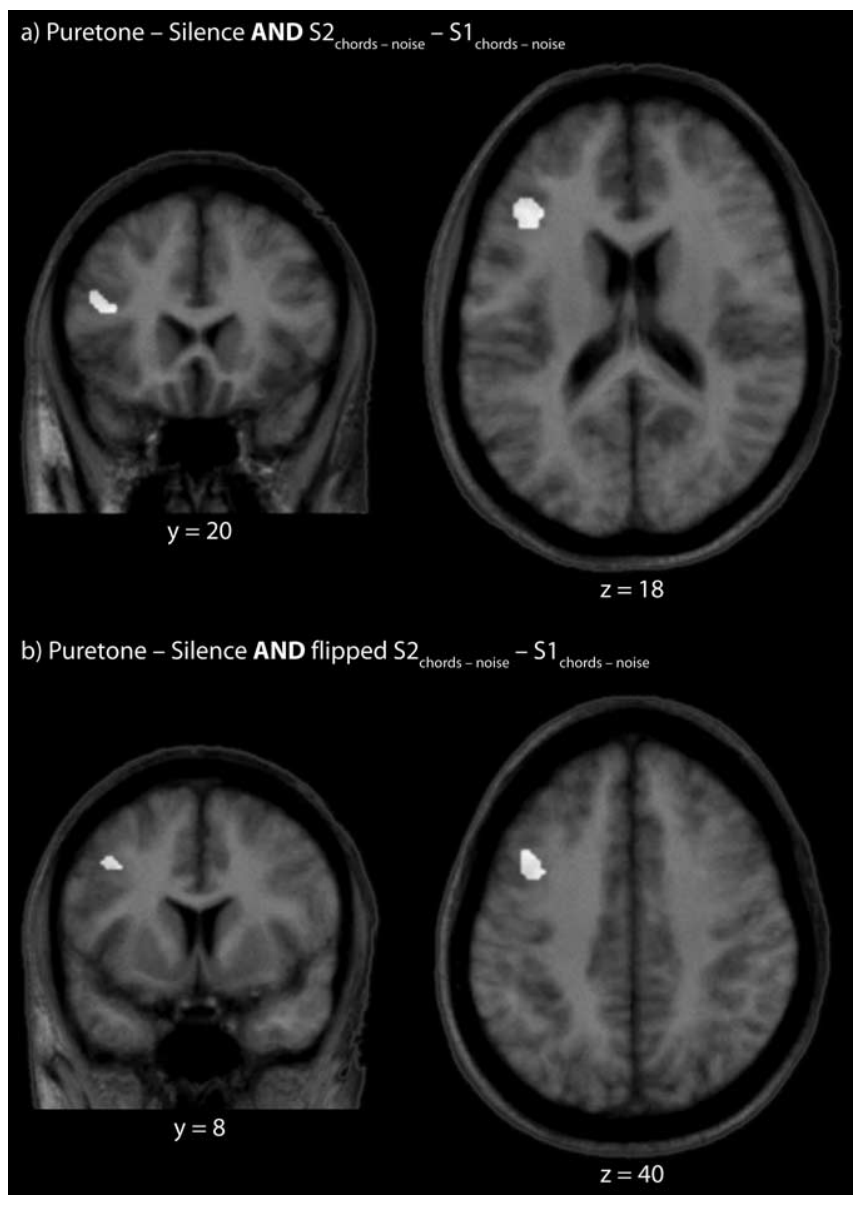

Figure 4. a, Spatial conjunction of the tones - noise contrast in AP subjects from the PET results of Zatorre et al. (1998) and the $S 2_{\text {chords }}$ - noise $-S 1_{\text {chords }- \text { noise }}$ contrast of this study. $\boldsymbol{b}$, Same as $\boldsymbol{a}$ but with the $S 2_{\text {chords }- \text { noise }}-S 1_{\text {chords }}$ - noise image flipped at approximately the midsagittal line (see Results, Imaging: conjunction).

2001). Such roles are consistent with the nature of our task. These areas were also found to be active in the results of Zatorre et al.(1998).

\section{Conclusion}

The functional results described above paint a coherent picture of the retrieval of a conditional association between an auditory stimulus and an arbitrary label, which, in effect, is what happens when AP musicians name a note. The critical and defining feature of prototypical absolute-pitch musicians is that they were able to form long-term memory traces for the fundamental frequencies of a relatively large number of notes. There is no need to posit AP-specific mechanisms for the retrieval of that information and its association with verbal labels. Therefore, one interesting aspect of the hypothesis put forth in this investigation is that part of the chain of processing involved in absolute-pitch identification, the conditional associative pairing of a stimulus dimension to a label and the retrieval of this information, is a universal ability, which is merely applied specifically in the case of AP (pitch chroma to note name). This does not change the fact that those possessing AP have managed to form long-term representations of pitches with semitone resolution, which most, even rigorously trained, musicians have not. This high-resolution category formation in the auditory domain is the principal feature of the AP ability. Our experiment does not address this question. The AP ability is best described multidimensionally and must involve many aspects of cerebral function, some universal and some, perhaps, not. In conjunction with the results of Zatorre et al. (1998), it is interesting to note that the cognitive requirements of one of the latter stages of AP identification can be shown to have common neural substrates in AP musicians, non-AP musicians, and nonmusicians alike.

\section{References}

Amiez C, Kostopoulos P, Champod AS, Collins L, Petrides M (2005) Role of the premotor cortex during the performance of conditional visuo-motor associations: a block-design fMRI study. Hum Brain Mapp 1214.

Bachem A (1937) Various types of absolute pitch. J Acoust Soc Am 9:146-151.

Baharloo S, Johnston PA, Service SK, Gitschier J, Freimer NB (1998) Absolute pitch: an approach for identification of genetic and nongenetic components. Am J Hum Genet 62:224-231.

Belin P, Zatorre RJ, Hoge R, Evans AC, Pike B (1999) Event-related fMRI of the auditory cortex. NeuroImage 10:417-429.

Boettiger CA, D’Esposito M (2005) Frontal networks for learning and executing arbitrary stimulus-response associations. J Neurosci 25:2723-2732.

Cadoret G, Pike GB, Petrides M (2001) Selective activation of the ventrolateral prefrontal cortex in the human brain during active retrieval processing. Eur J Neurosci 14:1164-1170.

Cansino S, Williamson SJ (1997) Neuromagnetic fields reveal cortical plasticity when learning an auditory discrimination task. Brain Res 764:53-66.

Collins DL, Neelin P, Peters TM, Evans AC (1994) Automatic 3D intersubject registration of MR volumetric data in standardized Talairach space. J Comput Assist Tomogr 18:192-205.

Crowne DP, Dawson KA, Richardson CM (1989) Unilateral periarcuate and posterior parietal lesions impair conditional position discrimination learning in the monkey. Neuropsychologia 27:1119-1127.

Deiber MP, Wise SP, Honda M, Catalan MJ, Grafman J, Hallett M (1997) Frontal and parietal networks for conditional motor learning: a positron emission tomography study. J Neurophysiol 78:977-991.

Göbel SM, Johansen-Berg H, Behrens T, Rushworth MF (2004) Responseselection-related parietal activation during number comparison. J Cogn Neurosci 16:1536-1551.

Grafton ST, Fagg AH, Arbib MA (1998) Dorsal premotor cortex and conditional movement selection: a PET functional mapping study. J Neurophysiol 79:1092-1097.

Halsband U, Freund HJ (1990) Premotor cortex and conditional motor learning in man. Brain 113:207-222.

Halsband U, Passingham RE (1985) Premotor cortex and the conditions for movement in monkeys (Macaca fascicularis). Behav Brain Res 18:269-277.

Hantz E, Crummer G, Wayman J, Walton J, Frisina R (1992) Effects of musical training and absolute pitch on the neural processing of melodic intervals: a P300 event-related potential study. Music Perception 10:25-42.

Jäncke L, Gaab N, Wustenberg T, Scheich H, Heinze HJ (2001) Short-term functional plasticity in the human auditory cortex: an fMRI study. Brain Res Cogn Brain Res 12:479-485.

Keenan JP, Thangaraj V, Halpern AR, Schlaug G (2001) Absolute pitch and planum temporale. NeuroImage 14:1402-1408.

Klein M, Coles MGH, Donchin E (1984) People with absolute pitch process tones without producing a P300. Science 223:1306-1309.

Kostopoulos P, Petrides M (2003) The mid-ventrolateral prefrontal cortex: insights into its role in memory retrieval. Eur J Neurosci 17:1489-1497.

Koyama M, Hasegawa I, Osada T, Adachi Y, Nakahara K, Miyashita Y (2004) Functional magnetic resonance imaging of macaque monkeys performing visually guided saccade tasks: comparison of cortical eye fields with humans. Neuron 41:795-807.

Kurata K, Tsuji T, Naraki S, Seino M, Abe Y (2000) Activation of the dorsal premotor cortex and pre-supplementary motor area of humans during an auditory conditional motor task. J Neurophysiol 84:1667-1672.

Lepage M, Brodeur M, Bourgouin P (2003) Prefrontal cortex contribution to associative recognition memory in humans: an event-related functional magnetic resonance imaging study. Neurosci Lett 346:73-76.

Mazziotta J, Toga A, Evans A, Fox P, Lancaster J, Zilles K, Woods R, Paus T, Simpson G, Pike B, Holmes C, Collins L, Thompson P, MacDonald D, Iacoboni M, Schormann T, Amunts K, Palomero-Gallagher N, Geyer S, Parsons L, et al. (2001) A four-dimensional probabilistic atlas of the human brain. J Am Med Inform Assoc 8:401-430.

Morris JS, Friston KJ, Dolan RJ (1998) Experience-dependent modulation 
of tonotopic neural responses in human auditory cortex. Proc R Soc Lond B Biol Sci 265:649-657.

Nichols T, Brett M, Andersson J, Wager T, Poline JB (2005) Valid conjunction inference with the minimum statistic. NeuroImage 25:653-660.

Nobre AC (2001) The attentive homunculus: now you see it, now you don't. Neurosci Biobehav Rev 25:477-496.

Owen AM, Milner B, Petrides M, Evans AC (1996) Memory for object features versus memory for object location: a positron-emission tomography study of encoding and retrieval processes. Proc Natl Acad Sci USA 93:9212-9217.

Paus T (1996) Location and function of the human frontal eye-field: a selective review. Neuropsychologia 34:475-483.

Penhune VB, Zatorre RJ, MacDonald JD, Evans AC (1996) Interhemispheric anatomical differences in human primary auditory cortex: probabilistic mapping and volume measurement from magnetic resonance scans. Cereb Cortex 6:661-672.

Petrides M (1985) Deficits in non-spatial conditional associative learning after periarcuate lesions in the monkey. Behav Brain Res 16:95-101.

Petrides M (1986) The effect of periarcuate lesions in the monkey on the performance of symmetrically and asymmetrically reinforced visual and auditory go, no-go tasks. J Neurosci 6:2054-2063.

Petrides M (1990) Nonspatial conditional learning impaired in patients with unilateral frontal but not unilateral temporal lobe excisions. Neuropsychologia 28:137-149.

Petrides M (1997) Visuo-motor conditional associative learning after frontal and temporal lesions in the human brain. Neuropsychologia 35:989-997.

Petrides M, Pandya D (1994) Comparitive architectonic analysis of the human and the macaque frontal cortex. In: Handbook of neuropsychology (Boller F, Grafman J, eds), pp 17-58. Amsterdam: Elsevier Science.

Petrides M, Alivisatos B, Evans AC, Meyer E (1993a) Dissociation of human mid-dorsolateral from posterior dorsolateral frontal cortex in memory processing. Proc Natl Acad Sci USA 90:873-877.

Petrides M, Alivisatos B, Meyer E, Evans AC (1993b) Functional activation of the human frontal cortex during the performance of verbal working memory tasks. Proc Natl Acad Sci USA 90:878-882.

Pierrot-Deseilligny C, Milea D, Muri RM (2004) Eye movement control by the cerebral cortex. Curr Opin Neurol 17:17-25.

Podbros LZ, Stamm JS, Denaro FJ (1980) Associative function of the arcuate segment of the monkey's prefrontal cortex. Physiol Behav 24:103-109.

Price CJ, Friston KJ (1997) Cognitive conjunction: a new approach to brain activation experiments. NeuroImage 5:261-270.
Rizzolatti G, Luppino G (2001) The cortical motor system. Neuron 31:889-901.

Rosano C, Sweeney JA, Melchitzky DS, Lewis DA (2003) The human precentral sulcus: chemoarchitecture of a region corresponding to the frontal eye fields. Brain Res 972:16-30.

Rubia K, Russell T, Overmeyer S, Brammer MJ, Bullmore ET, Sharma T, Simmons A, Williams SC, Giampietro V, Andrew CM, Taylor E (2001) Mapping motor inhibition: conjunctive brain activations across different versions of go/no-go and stop tasks. NeuroImage 13:250-261.

Rushworth MF, Nixon PD, Passingham RE (1997) Parietal cortex and movement. I. Movement selection and reaching. Exp Brain Res 117:292-310.

Rushworth MF, Krams M, Passingham RE (2001) The attentional role of the left parietal cortex: the distinct lateralization and localization of motor attention in the human brain. J Cogn Neurosci 13:698-710.

Schneider P, Scherg M, Dosch HG, Specht HJ, Gutschalk A, Rupp A (2002) Morphology of Heschl's gyrus reflects enhanced activation in the auditory cortex of musicians. Nat Neurosci 5:688-694.

Stamm JS (1973) Functional dissociation between the inferior and arcuate segments of dorsolateral prefrontal cortex in the monkey. Neuropsychologia 11:181-190.

Takeuchi AH, Hulse SH (1993) Absolute pitch. Psychol Bull 113:345-361.

Teismann IK, Soros P, Manemann E, Ross B, Pantev C, Knecht S (2004) Responsiveness to repeated speech stimuli persists in left but not right auditory cortex. NeuroReport 15:1267-1270.

Ward WD (1999) Absolute pitch. In: The psychology of music, Ed 2 (Deutsch D, ed), pp 265-298. San Diego: Academic.

Wayman JW, Frisina RD, Walton JP, Hantz EC, Crummer GC (1992) Effects of musical training and absolute pitch ability on event-related activity in response to sine tones. J Acoust Soc Am 91:3527-3531.

Worsley KJ, Liao CH, Aston J, Petre V, Duncan GH, Morales F, Evans AC (2002) A general statistical analysis for fMRI data. NeuroImage 15:1-15.

Zatorre RJ (2003) Absolute pitch: a model for understanding the influence of genes and development on neural and cognitive function. Nat Neurosci 6:692-695.

Zatorre RJ, Perry DW, Beckett CA, Westbury CF, Evans AC (1998) Functional anatomy of musical processing in listeners with absolute pitch and relative pitch. Proc Natl Acad Sci USA 95:3172-3177.

Zatorre RJ, Belin P, Penhune VB (2002) Structure and function of auditory cortex: music and speech. Trends Cogn Sci 6:37-46. 